\title{
Ground Defected Planar Super-wideband Antenna: A Suitable Transceiver for Short Distance Wireless Communication
}

\author{
Rezaul Azim* \& A. K. M. Ariful Haque Siddique \\ Department of Physics, University of Chittagong, Bangladesh
}

\begin{abstract}
A planar microstrip patch super-wideband antenna is presented for short distance wireless communication applications. The antenna is comprised of a simple patch and a ground plane and etched on two sides of a 1.6 mm-thick standard FR4 substrate material with a relative permittivity of 4.5 and loss tangent (0.02). The proposed antenna possesses a compact size of $29 \times 20.5 \mathrm{~mm}^{2}$ with an electrical dimension of $0.25 \lambda \times 0.18 \lambda$. To enhance the operating bandwidth, the ground plane is modified by adding seven small rectangular slots on its upper side. Through numerical studies, it is found that insertion of the slots enhances the coupling between the patch and ground plane resulting in achievement of a super-wide operating band. From the measurements, it is observed that the fabricated prototype antenna has a bandwidth from 2.63 to more than $18 \mathrm{GHz}$, a symmetric omnidirectional radiation characteristic and the maximum peak gain of $5.85 \mathrm{dBi}$ which makes it a suitable transceiver for short distance communication applications.
\end{abstract}

Keywords: Antenna; defected ground plane; microstrip patch; transceiver; wireless communication

\section{INTRODUCTION}

The advent of the new information era has ushered in a new dimension in wireless communication technology, which has significantly influenced and benefited the human life. It has been elevated to a whole new level by the commissioning of new technologies such as mobile and satellite networks. The proliferation of communication systems has driven the wireless technology to the verge of a revolutionary wireless communications. Replete with the cutting-edge technologies, in recent years the wireless industry has revealed a significant growth. This tremendous development is due to the phenomenal advances in the ubiquitous wireless connectivity. As a transceiver, antenna plays the key role to benefit huge subscribers and to cater data with greater data transmission rates. To be a suitable transceiver, an antenna has to be of low profile, convenient weight, low cost and congenial to the design of the mounting devices. Moreover, antennas should be able to demonstrate a good impedance matching to achieve wide operating bandwidth, stable radiation patterns, satisfactory gain and efficiency.

Short distance wireless communication systems, such as wireless local area network (WLAN), wireless personal area network (WPAN), wireless body area network (WBAN), wireless fidelity (WiFi), world interoperability for microwave access (WiMAX), wireless local area network (WLAN), ground penetrating radars, medical imaging system even robotics need very high spectral efficiency through the use of appropriate technical standards. In all these applications, the antenna is the most critical part which determines whether the system will be practical and successful one in future. Good numbers of studies have been reported to investigate antennas for super-wideband wireless communication. For example, a wideband stacked patch antenna is presented by Li et al. (2012) where capacitively coupled feeding is used to attain broadband characteristics. Using a double layer radiator comprising of two pairs of feeding probes connected to the feeding structure, the designed antenna with a diameter of 160 $\mathrm{mm}$ achieved a bandwidth from 1.1-1.7 GHz. A multilayer patch antenna where broadband nature is achieved by stacking a patch over fed patch was reported by Ansari et al. (2012). Using a three layer patch, the proposed antenna achieved a bandwidth of $42.6 \%$. The designs proposed by Li et al. (2012), Ansari et al. (2012) and Li \& Behdad (2017) have three dimensional (3D) structures that feature with high gain, directional radiation pattern but not suitable to be integrated with other microwave circuitries due to their 3D profile and large volumetric size.

Comparing with 3D antennas such as stacked patch, log periodic, horn, spiral structured antennas, the planar antenna appears to be the most coveted choice having the hallmarks of small, thin, low profile and easily incorporable with portable wireless devices (Hussine et al. 2012). This antenna can be made on a portion of standard $\mathrm{PCB}$ and its performances in wideband communication determine the success of high speed communication systems. For example, for ultra-wideband communication, a dipole antenna was recently proposed by Nazli et al. (2010). By inserting elliptical slots on the arms of a dipole, the prototype attained a wide impedance bandwidth of $94.4 \%$. But this design has a large volumetric size of $106 \times 85 \mathrm{~mm}^{2}$ which may restrict it use in many portable devices. In (Alsath \& Kanagasabai 2015), a compact ultra-wideband antenna was presented for automotive communication application. The bandwidth of the designed antenna is enhanced by modifying the ground plane with the addition of an extended ground stub and achieved 
an impedance band ranging from 3.1 - $10.9 \mathrm{GHz}$. For ultrawideband application, a $41 \times 41 \mathrm{~mm}^{2}$ size planar wearable antenna was presented by Ali et al. (2016). The layout of this design comprises of a circular patch and a defected rectangular ground structure and achieved a bandwidth of 11.4 GHz. In 2016, Tran and Park propose a circular polarized resonant cavity antenna with high gain characteristics (Tran \& Park 2016). In this design, a planar conducting screen ground plane was placed below the patch and a superstrate was placed in the front side of the patch and the optimized design achieved a fractional bandwidth of $40 \%(5 \cdot 6-8 \cdot 4 \mathrm{GHz})$. However, a circularly polarized radiation pattern limits its application in many devices.

Different methods such as the use of the thick substrate, patches with multiple layers, insertion of slots/slits, use of metamaterial and modification of radiator shape have also been observed to increases the bandwidth of microstrip antenna. The defection of the ground plane is another remarkable method to widen the operating band of the planar antenna (Azim et al. 2011a; Azim et al. 2011b; Ghosh et al. 2016; Azim et al. 2013a). It not only widens the operating band but also reduces the cross-polarized field. For example, to widen the operating band, the upper side of the ground structure of antenna presented by Azim et al. (2011a) was reshaped to a saw-tooth type and the antenna with new ground plane attained $43.6 \%$ more bandwidth than the original one. To enhance the bandwidth, the ground plane of the antenna reported in (Azim et al. 2011b) was modified by inserting a rectangular slot of dimension $6 \times 0.9 \mathrm{~mm}^{2}$. To achieve a bandwidth of $3.1 \mathrm{GHz}$, a pair of complementary slots was inserted on the ground plane of the design proposed by Ghosh et. al (2016). Though many of these reported antennas exhibit a wide operating band, some possess big sizes that limit their integration in portable devices while others possess complex layout resulting in cost ineffective for much production.

In this paper, a planar antenna is presented for short distance wireless communication applications. The radiating element and the ground plane are etched on both sides of a double-sided FR4 dielectric material and is fed by a microstrip feed line of $50 \Omega$ characteristics impedance. To attain wideband characteristics, the larger ground plane that covers the whole substrate area is shrunken to partial one with a side length of $5 \mathrm{~mm}$. It is found that, compared to the whole ground plane, the design with a smaller ground plane achieved $131.5 \%$ wider operating bandwidth. To widen the operating bandwidth further, a total of seven small slots have been added to the top edge of the smaller ground plane. If the antenna is treated as a resonating element, the insertion of slots alters its inductance resulting in shifting of first resonance mode towards the lower frequency spectrum. Therefore, the designed antenna achieved a super-wide bandwidth $(2.85$ $\mathrm{GHz}$ to around $18 \mathrm{GHz}$ ) due to proper impedance matching in between ground plane and patch.

\section{DESCRIPTION OF THE ANTENNA}

Figure 1 presents the structure and design dimension of the proposed antenna. The radiating element with a dimension of $L_{\mathrm{P}} \times W_{\mathrm{P}}$ and ground plane with a side length, $L_{\mathrm{G}}$ are etched on both sides of a standard FR4 dielectric material of $\varepsilon_{\mathrm{r}}=$ 4.5 , loss tangent, $\tan \delta=0.02$ and $h_{\mathrm{SUB}}=1.6 \mathrm{~mm}$. To feed the antenna with a Sub-Miniature version A (SMA) connector, a microstrip line with length $l_{\mathrm{f}}$ and width $w_{\mathrm{f}}$ is designed. The feedline is $6.4 \mathrm{~mm}$ away from the left edge of the substrate and characterized with $50 \Omega$ characteristics impedance. The distance between the radiator and the ground plane is $g$. The overall dimension of the proposed antenna is $L_{\mathrm{SUB}} \times W_{\mathrm{SUB}}$.

To attain super-wideband characteristics, the ground plane structure of a planar microstrip antenna can suitably be selected. As the current in a planar antenna is flowed both on the patch and the ground plane, it is not possible to ignore the emission of electromagnetic radiation from ground plane. Therefore, the structure and dimension of the ground structure effectively influence the bandwidth which is demonstrated in Figure 2(a). It can be observed from the plot that, when the ground plane covers the whole substrate area, the proposed antenna exhibits the smallest bandwidth in comparison to the antennas with the partial ground plane and multi-slotted partial ground plane. The numerical voltage standing wave ratio (VSWR) value is drastically lowered when the ground plane is shrunken to part one and drops to below 2. Lastly, the introduction of seven slots in the upper side helps the optimized design to achieve a bandwidth of 15.14 $\mathrm{GHz}$. The insertion slots change the structure of the ground plane causes the change in capacitance and inductance of the input impedance, for which the operating bandwidth changes. The shape and size of the ground plane can control the resonance characteristics of the radiator because etching of slots influences the coupling between the radiator and slotted partial ground plane as the magnetic flux flow in the etched out aperture. It can also be noted from Figure 2(b) that insertion of seven slots in the ground plane helps the presented antenna to demonstrate six resonances at 3.3 $\mathrm{GHz}, 6.325 \mathrm{GHz}, 9 \mathrm{GHz}, 11 \mathrm{GHz}, 13 \mathrm{GHz}$, and $15.9 \mathrm{GHz}$ and overlapping of these resonances resulting in a very wide operating band with lower reflection coefficient. Though similar technique was reported in (Azim et al. 2011c), the proposed antenna achieved wider bandwidth than that due to the shape and size of the etched slots. When compared with the slots inserted in the antenna reported in (Azim et al. 2011c), the slots in the realized antenna possess rectangular shape and thus the current is uniformly distributed resulting in uniform change in antenna impedance. This change in impedance changes the effective permittivity of the antenna and hence the operating bandwidth is enhanced (Saad et al. 2011; Rahman et al. 2017). 


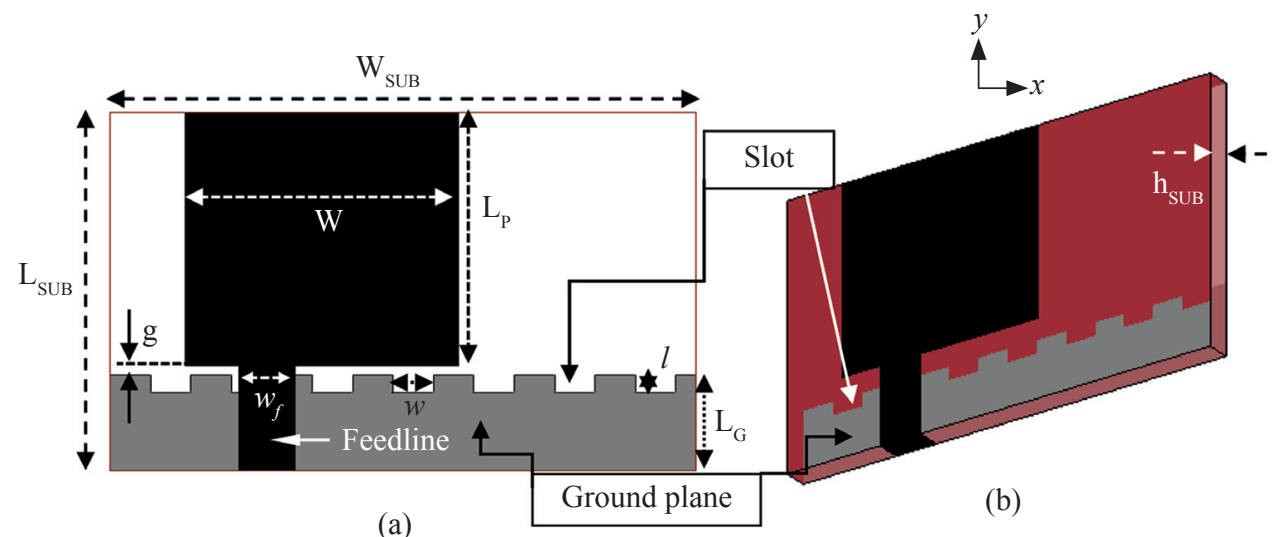

FIGURE 1. Proposed design with defected ground plane: (a) top view (b) perspective view. All parameters are in mm: $w=2, l=1$, $g=0.5, L_{\mathrm{G}}=5.5, w_{\mathrm{f}}=2.75, W_{\mathrm{P}}=13.5, L_{\mathrm{P}}=14.5, W_{\mathrm{SUB}}=29, L_{\mathrm{SUB}}=20.5$

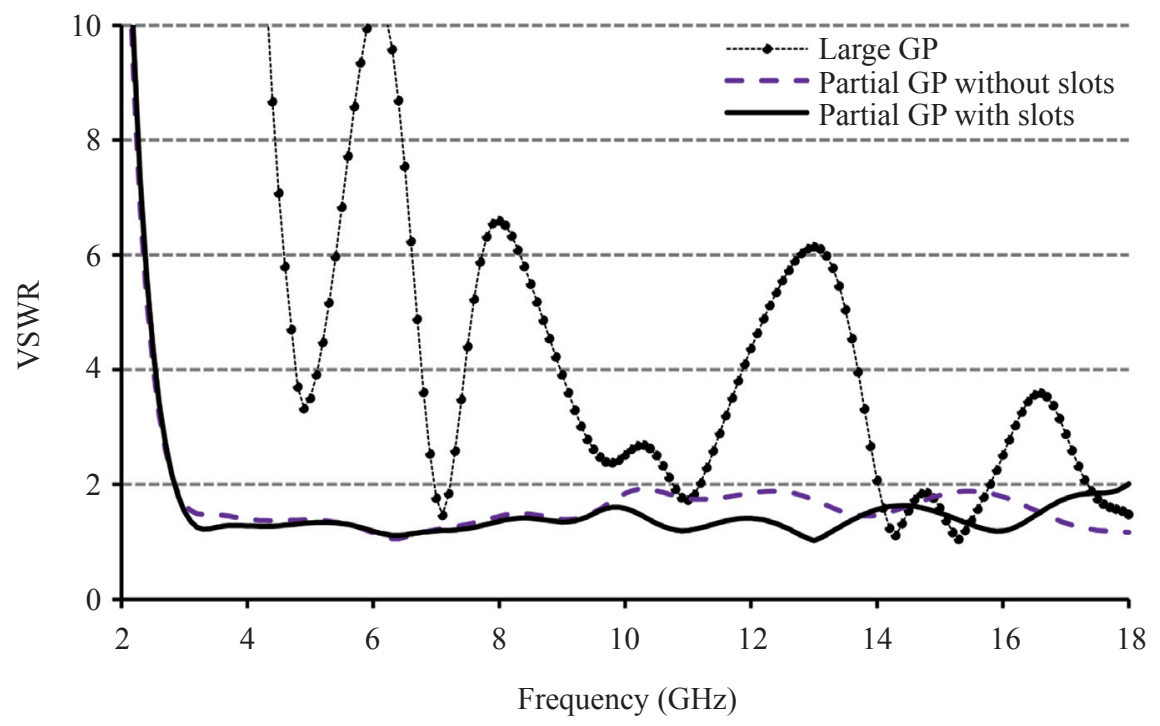

(a)

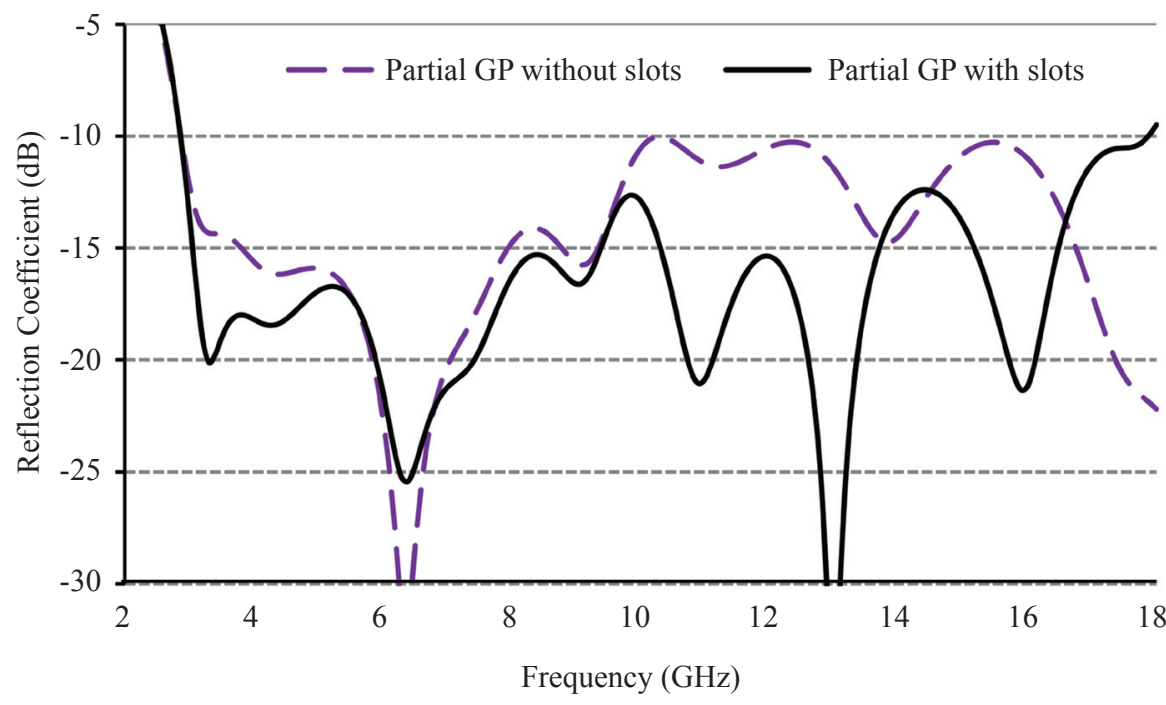

(b)

FIGURE 2. (a) VSWR's responses for different sizes of the ground plane (GP) and (b) reflection coefficient for the ground plane without and with slots 


\section{SYNTHETIC AND EXPERIMENTAL RESULTS}

The performance of the proposed design is analyzed by EM simulator Zeland IE3D. IE3D is based on an integral equation approach and method of moments. In the simulation, the meshing frequency is taken as $20 \mathrm{GHz}$, the cell per wavelength is 15 and the size of the grid cell is chosen as 0.001 . The meshing scheme is taken as contemporary and maximum layer distance is chosen as $0.0005 \mathrm{~mm}$. After the optimization, a pair of the prototype is fabricated for experimental verification, as depicted in Figure 3. A PNA series vector network analyzer (E8362C) from Keysight Technologies is used to measure the VSWR responses. The VSWR curves of the optimized design are displayed in Figure 4. It is evident from the figure that the fabricated design achieved a band from $2.63 \mathrm{GHz}$ to more than $18 \mathrm{GHz}$ for VSWR $\leq 2$ which makes it suitable to be used in WiMAX, WiFi, WLAN, UWB, C-band, X-band communication and microwave imaging applications. The slight deviation of the measured VSWR observed at around $14-16 \mathrm{GHz}$ band may be due to the mismatch at that band resulted from imperfect prototyping, the effect of feeding cable during measurement and high losses of FR4 dielectric material.

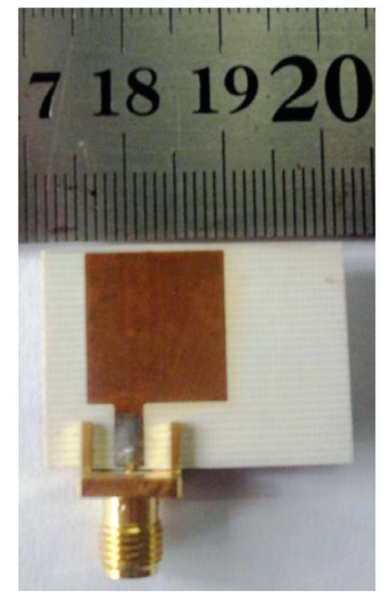

(a)

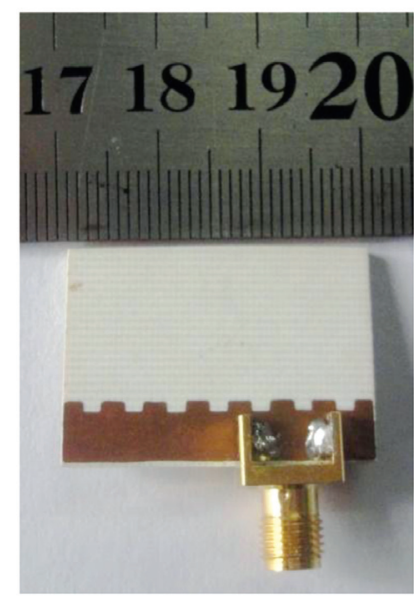

(b)
FIGURE 3. Prototype of the proposed antenna (a) top view and (b) bottom view

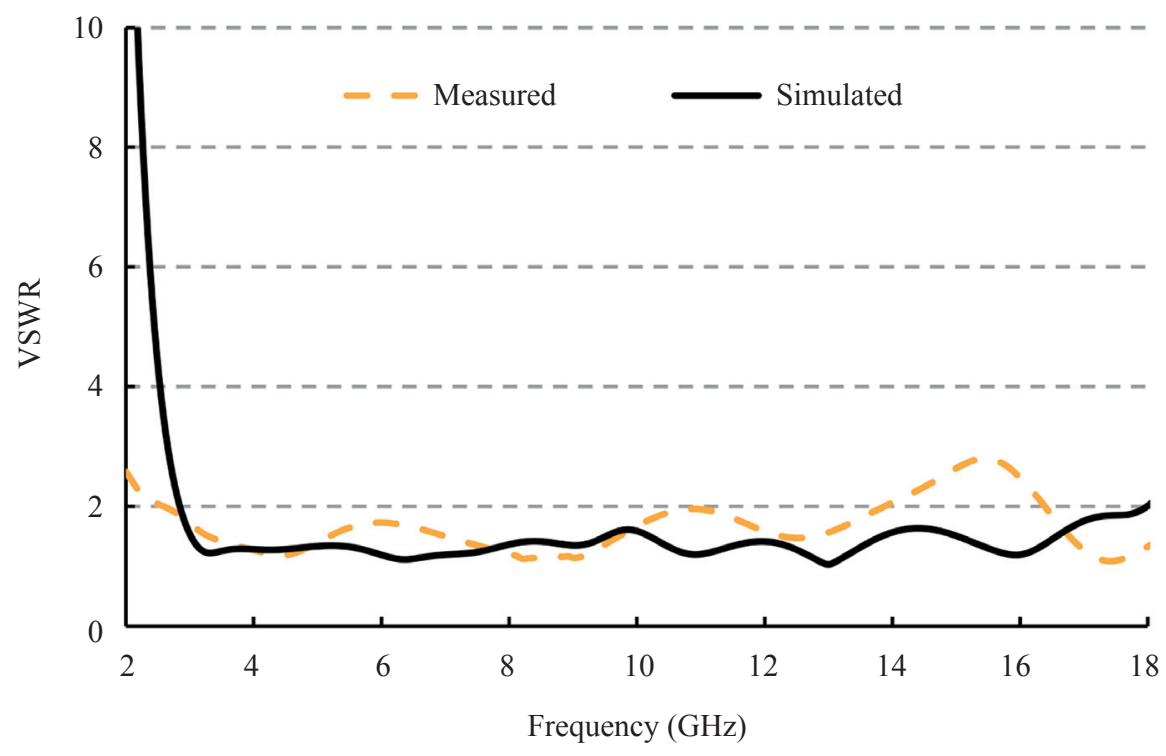

FIGURE 4. Measured and simulated VSWR's of the proposed antenna

Satimo's StarLab near-field antenna measurement system as shown in Figure 5 is used to measure the gain, efficiency and radiation pattern of the prototype. This system allows measuring the antenna's electric fields within the near-field region with an aim to compute the corresponding far-field values of the antenna under test (AUT). The AUT, placed on the test bed, is positioned in the middle of a circular "arch" that contains 16 individual measuring probes. These probes are placed at an equal distance surrounding the circular surface. The AUT is rotated horizontally in $360^{\circ}$ angle, and this rotation and array of probes together does a full 3D scan of AUT and collecting data for $3 \mathrm{D}$ radiation patterns. The far-field data is then employed to compute the gain and efficiency of the AUT.
Figure 6 displays the measured and simulated peak gain of the proposed antenna from where it can be mentioned that the measured data are reasonably agreed with the simulated one. From the gain curve, it can also be commented that the prototype has achieved an average gain of $3.36 \mathrm{dBi}$. The maximum measured peak gain is about $5.85 \mathrm{dBi}$. The measured radiation efficiency of the proposed design is presented in Figure 7 from where it is noticed that the peak efficiency of the fabricated prototype is $83.8 \%$ and the average efficiency is $60 \%$. It can also be observed that the efficiency of the proposed antenna is lower especially at high frequencies which may be due to the excitation of the higher order current modes. Moreover, as the antenna is designed on FR4 lossy material, it efficiency is lower at high frequency due to the high dielectric loss which can be improved by the use of loss free expensive dielectric substrate material. 


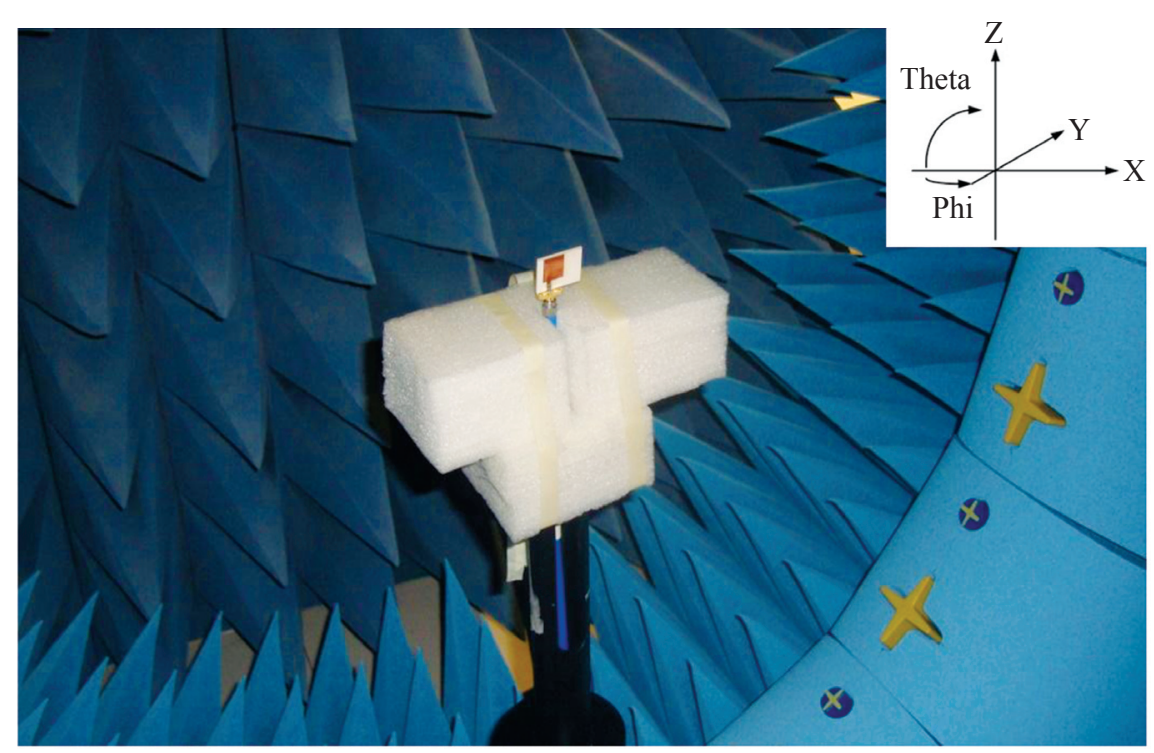

FIGURE 5. Radiation characteristics measurement setup in StarLab with coordinate system

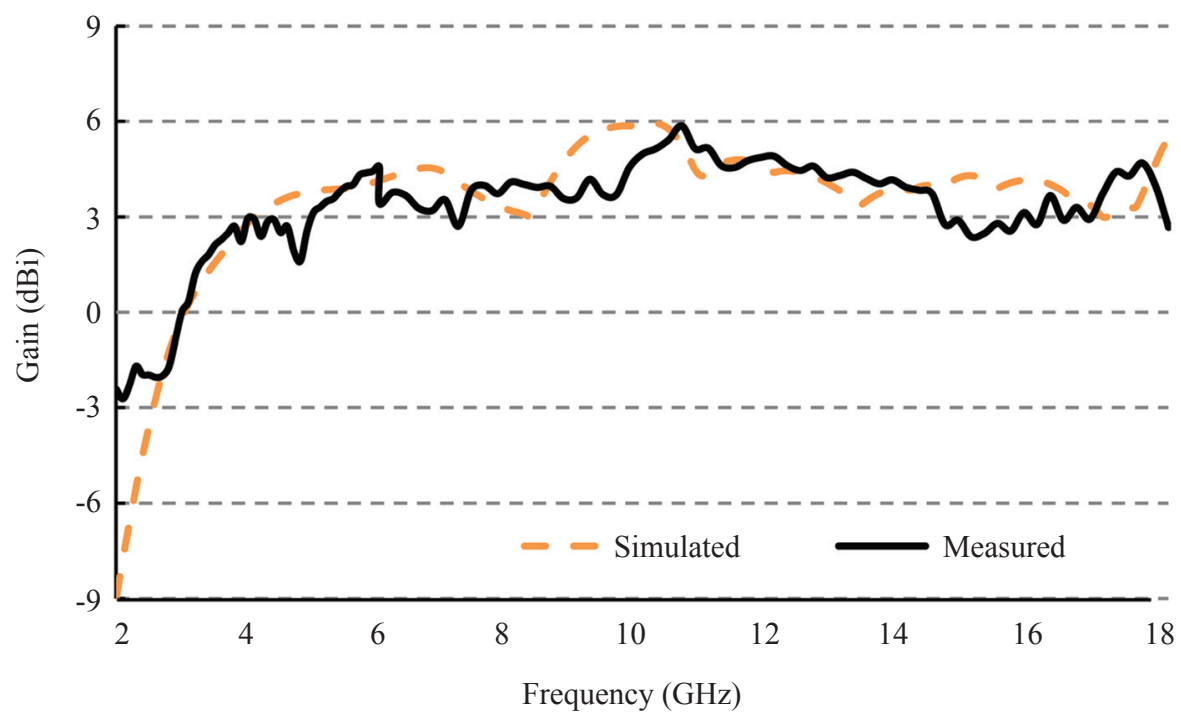

FIGURE 6. Measured and simulated gain of the proposed antenna

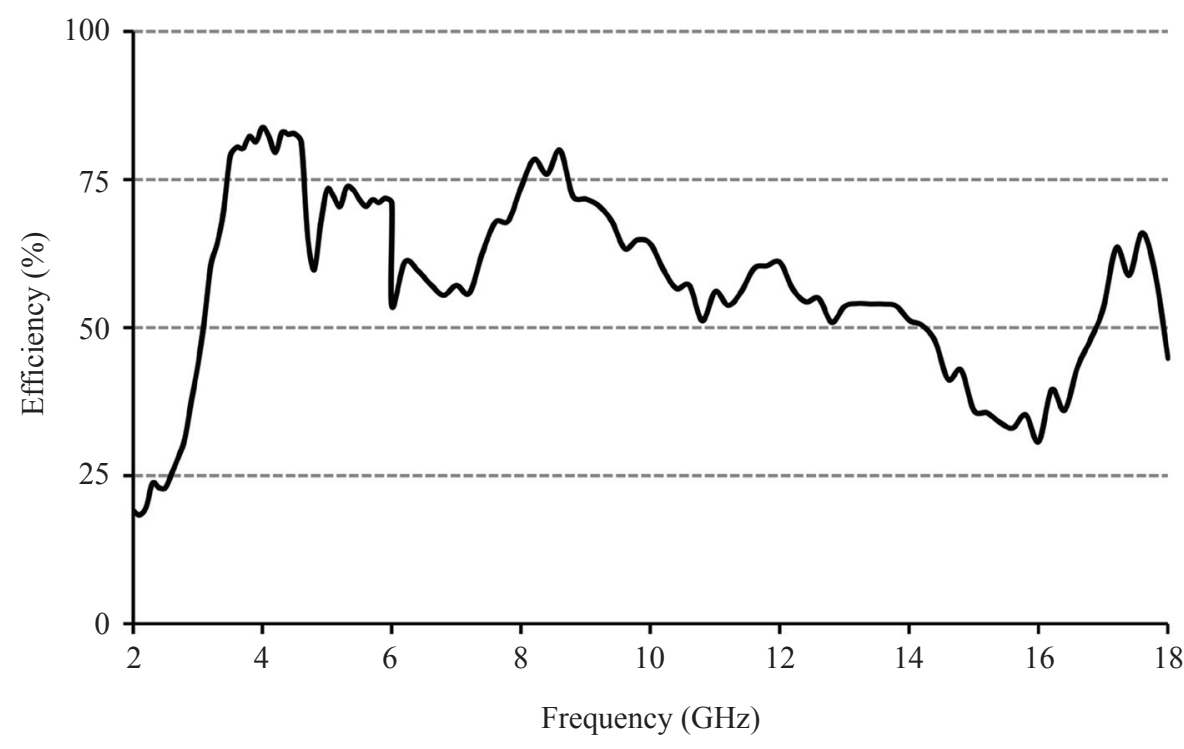

FIGURE 7. Measured efficiency of the proposed antenna 
The simulated current distributions on the surface of the presented antenna at six resonance frequencies of 3.3, 6.325, 9, 11, 13 and 15.9 GHz are depicted in Figures 8(a)(f) correspondingly, and the measured radiation patterns at these frequencies in $E$-and $H$-plane are shown in Figures 9(a)-(f). The radiation patterns are normalized with respect to their maximum value. It can be evident from the current distribution in Figure 8(a) that at $3.3 \mathrm{GHz}$, the current is evenly distributed in the patch and ground plane, hence the radiation pattern is omnidirectional in nature as shown in Figure 9(a). At second resonance frequency of 6.325 $\mathrm{GHz}$, the current in the patch is still evenly distributed as in Figure 8(b), and thus the antenna still approximately exhibits omnidirectional radiation characteristics as depicted in Figure 9(b). The current distribution pattern at third resonance at 9 $\mathrm{GHz}$ is depicted in Figure 8(c), thus indicating a third-order harmonic result and hence in the radiation pattern at $9 \mathrm{GHz}$ few nulls have been observed at broadside direction as in Figure 9(c). A slightly complicated current distribution at 11 GHz corresponding to fourth-order harmonic is illustrated in Figure 8(d) and radiation pattern at this frequency still able to retain its Omni directionality in $H$-plane as displayed in Figure 9(d). Figure 8(e) plots the current distribution at a higher frequency of $13 \mathrm{GHz}$ from where it is seen that higher order current modes are start developing, and the current is less symmetrically circulated on the patch. Therefore, the radiation patterns displayed in Figure 9(e) become slightly directional with the exhibition of some nulls. At a very

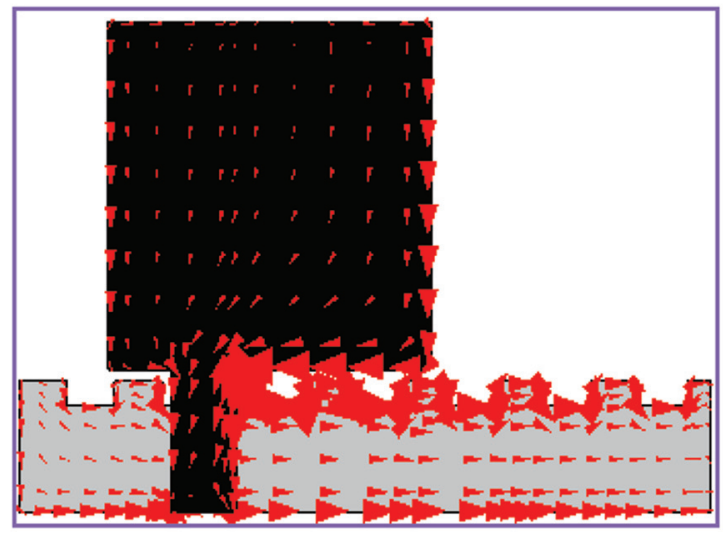

(a)

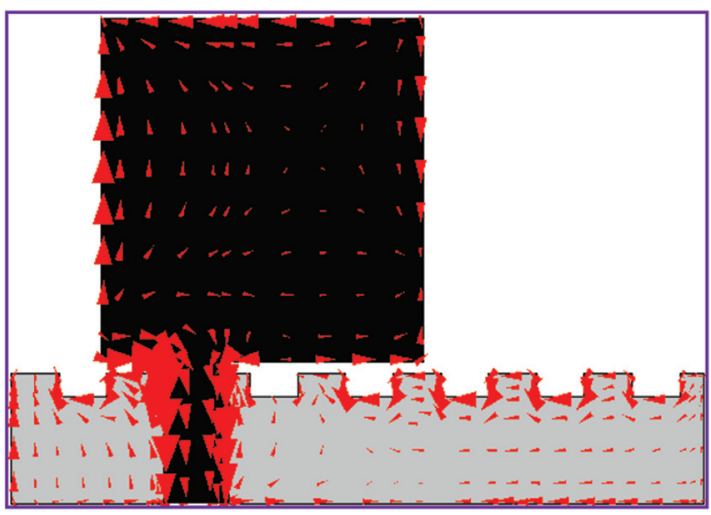

(c) higher frequency of $15.9 \mathrm{GHz}$, the surface current is no longer symmetrically distributed on the patch and ground plane since higher order modes are excited as displayed in Figure 8(f). The radiation patterns as in Figure 9(f) become directional with the introduction of more nulls. In Figure 8 , the current is mainly distributed along the feed line and patch. Moreover, the current is densely concentrated in the slots of ground plane which clearly demonstrates the effect of slots on antenna performances. From the plot, it is seen that as the frequency increases, the cross-polarization field become higher in comparison to the co-polarized one. This increment of the cross-polarization field may be due to the excitation of higher order current modes, especially at higher frequencies. Furthermore, as the substrate thickness of the proposed antenna is less than the quarter wavelength, an $180^{\circ}$ phase difference is introduced between the reflected surface waves and the one that impinges on the substrate. This $180^{\circ}$ out of phase reflected surface waves when combines with the radiating waves produce a destructive interference resulting in the increment of cross-polarization field and deterioration of antenna characteristic in terms of gain, efficiency and return loss. The increased cross-polarized field can be minimized by providing an in phase excitation of the surface waves as reported by Reddy et al. (2012). Despite higher crosspolarization field especially at higher frequencies, the results in Figures 9(a)-(f) illustrate that the radiation patterns of the anticipated antenna are robust over the entire the operating band.

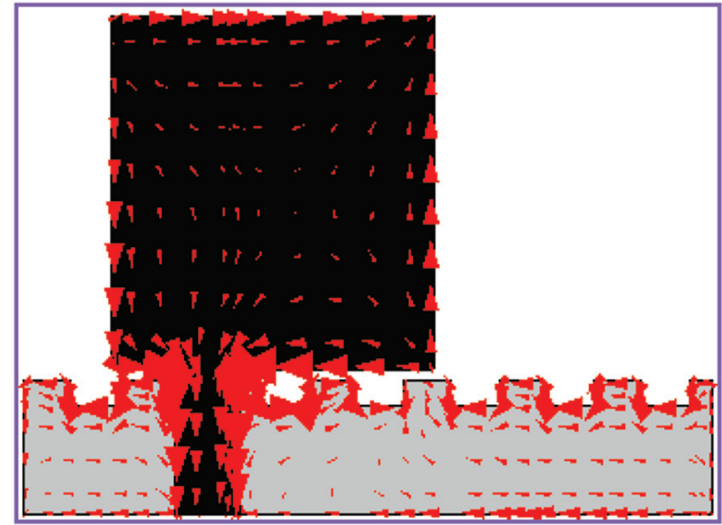

(b)

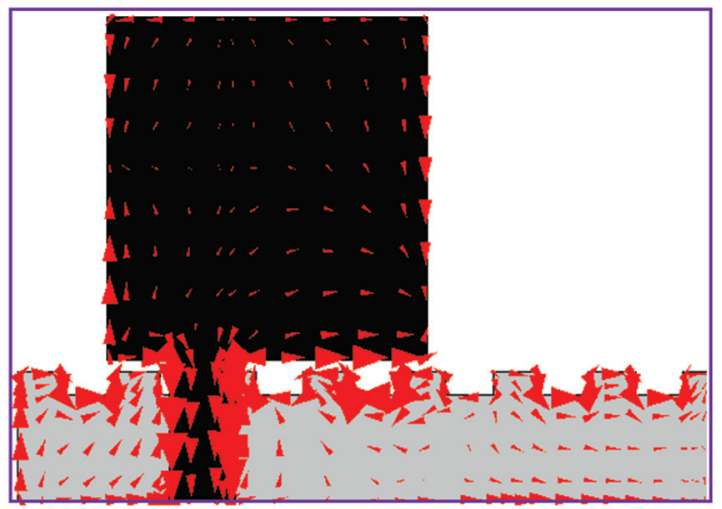

(d) 


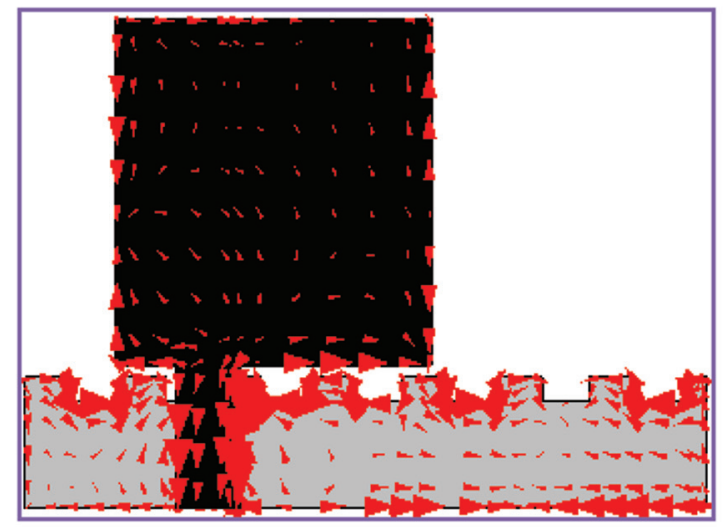

(e)

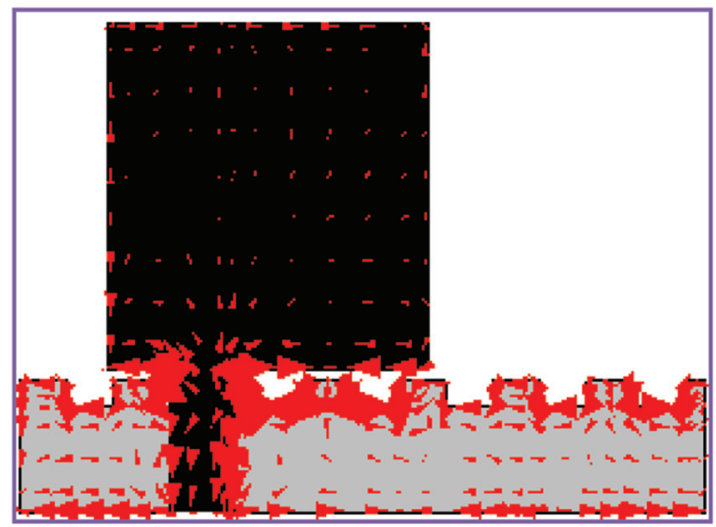

(f)

FIGURE 8. Simulated vector current distribution at (a) $3.3 \mathrm{GHz}$, (b) $6.325 \mathrm{GHz}$, (c) $9 \mathrm{GHz}$, (d) $11 \mathrm{GHz}$, (e) $13 \mathrm{GHz}$, and (f) $15.9 \mathrm{GHz}$
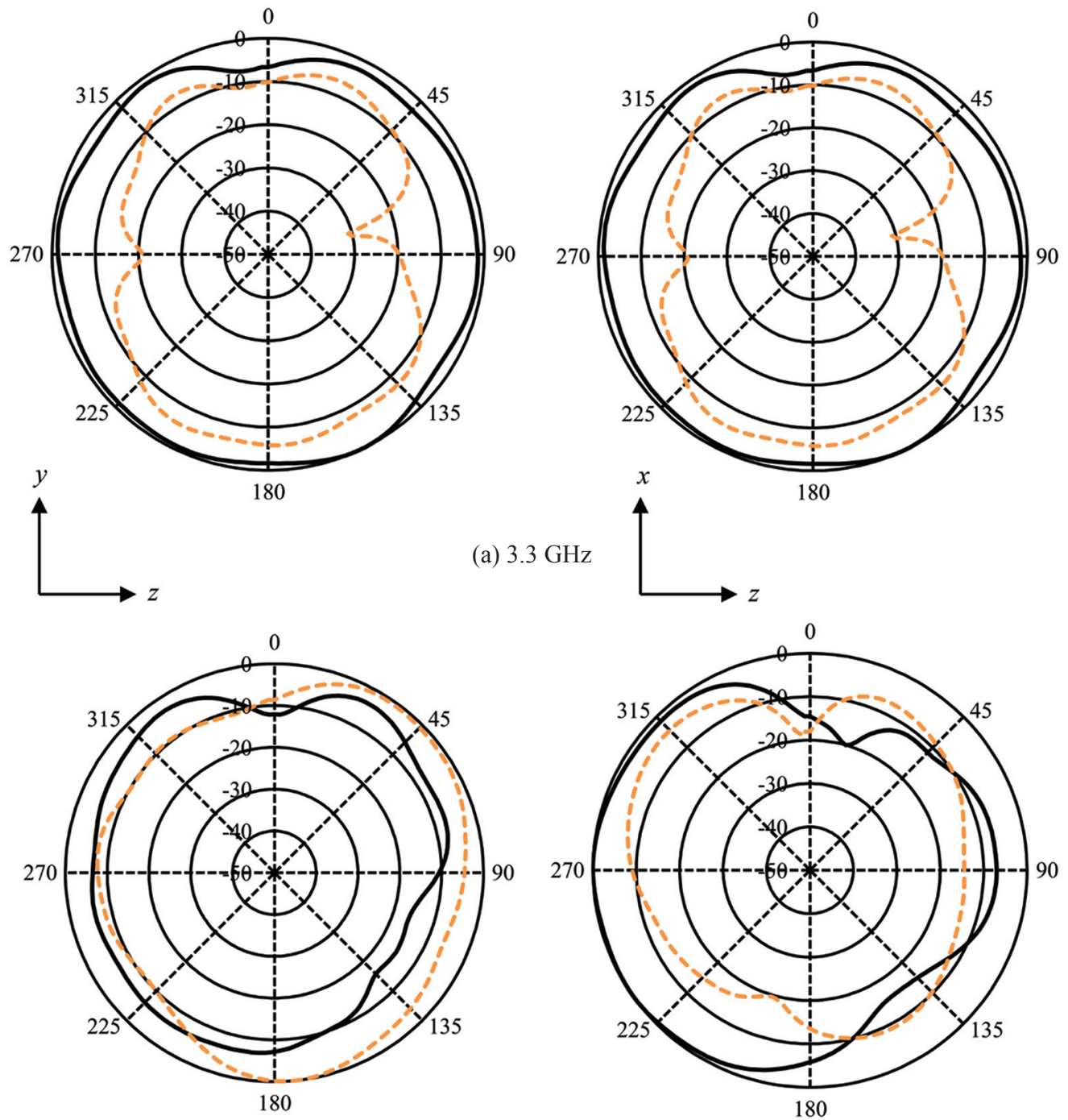

(b) $6.325 \mathrm{GHz}$ 

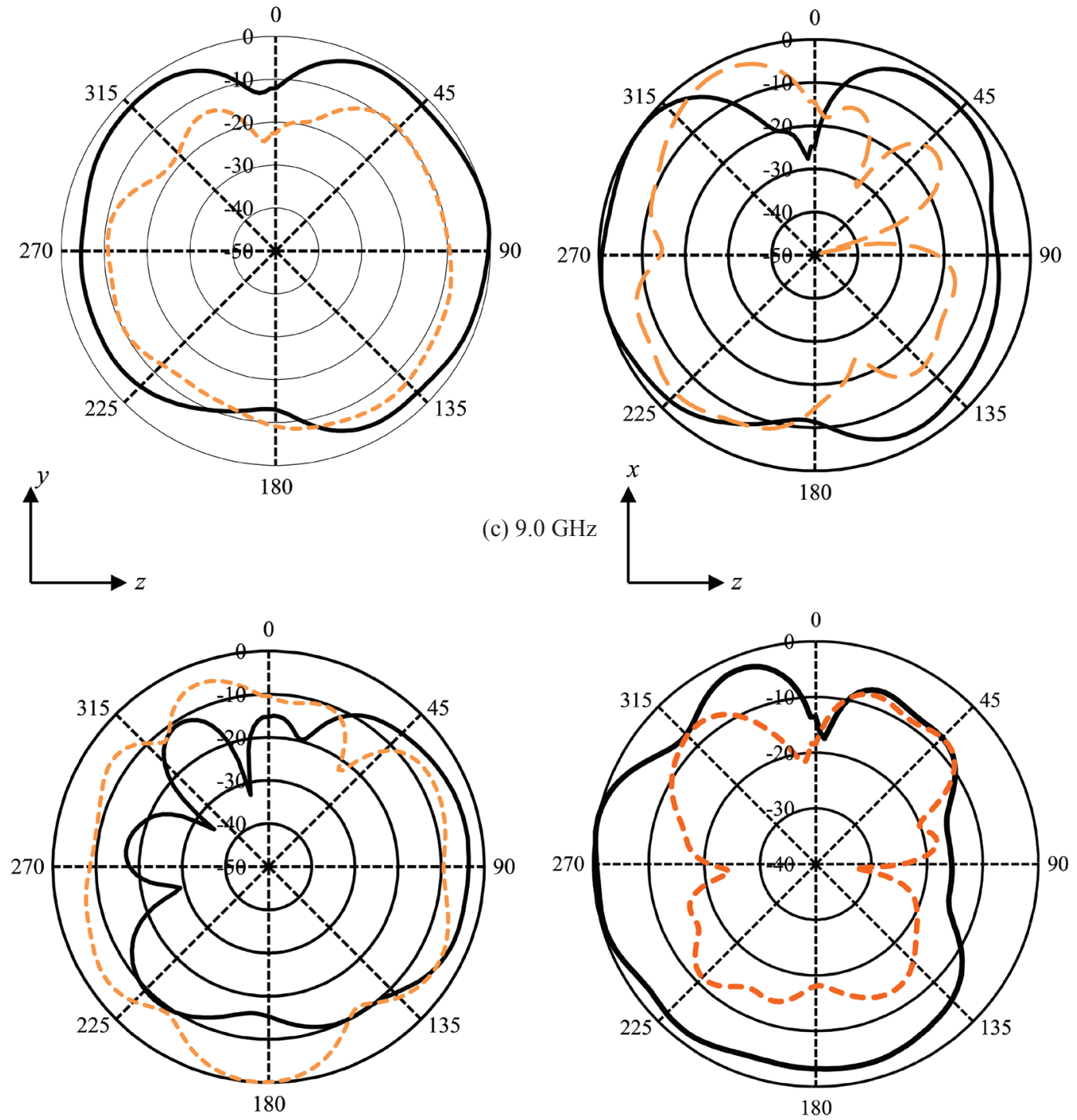

(d) $11 \mathrm{GHz}$
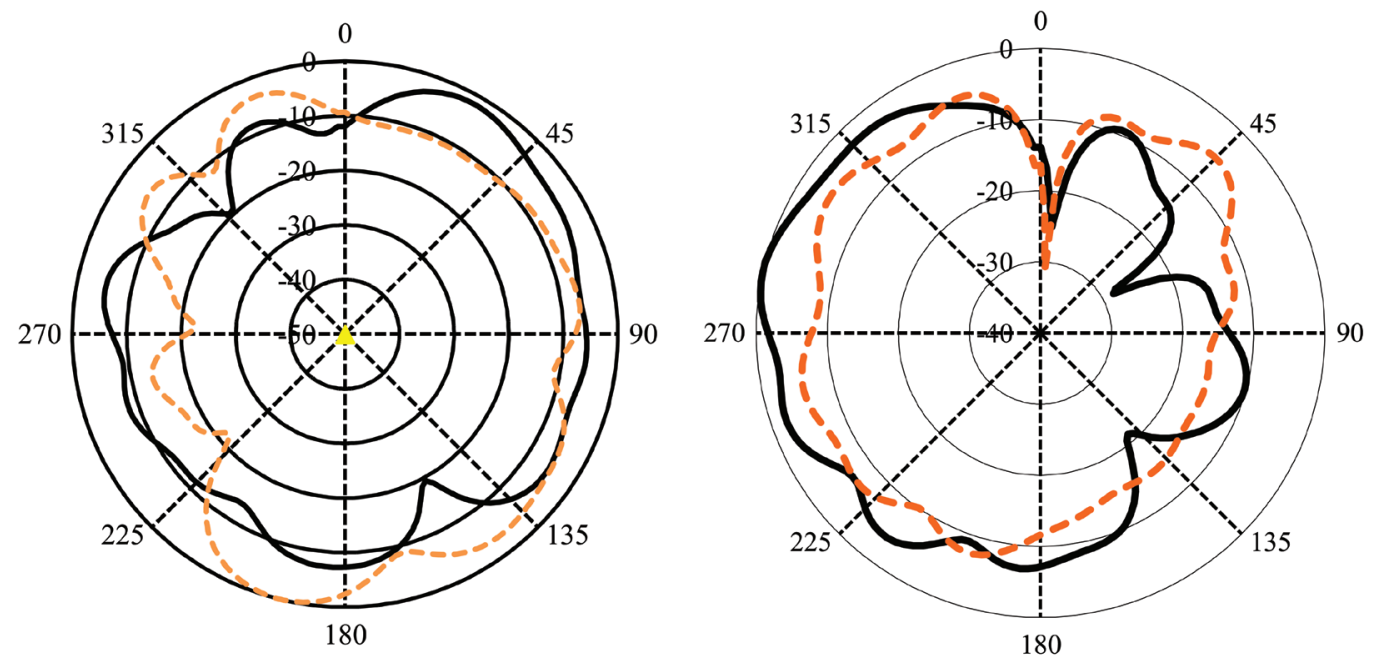

(e) $13 \mathrm{GHz}$ 

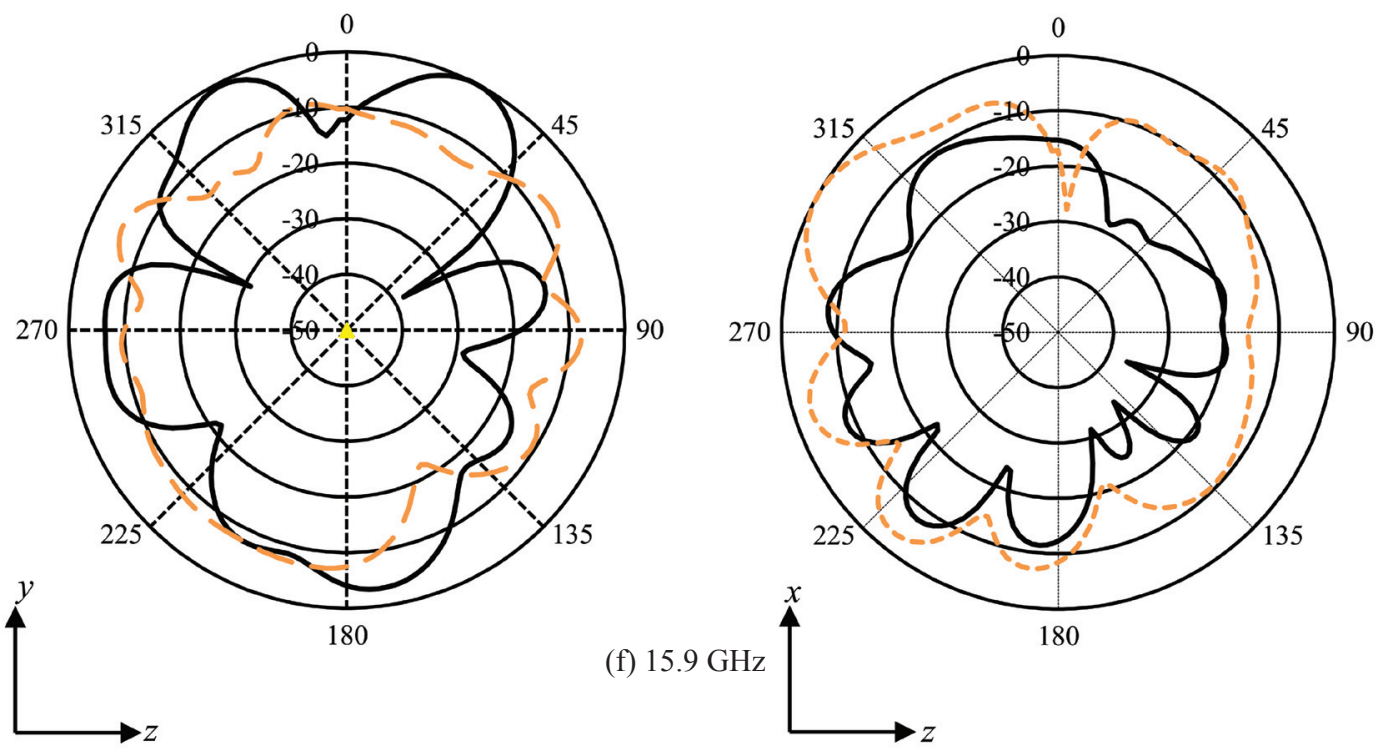

FIGURE 9. Measured radiation patterns at different frequencies

Co-polarization, - - - - - Cross-polarization)

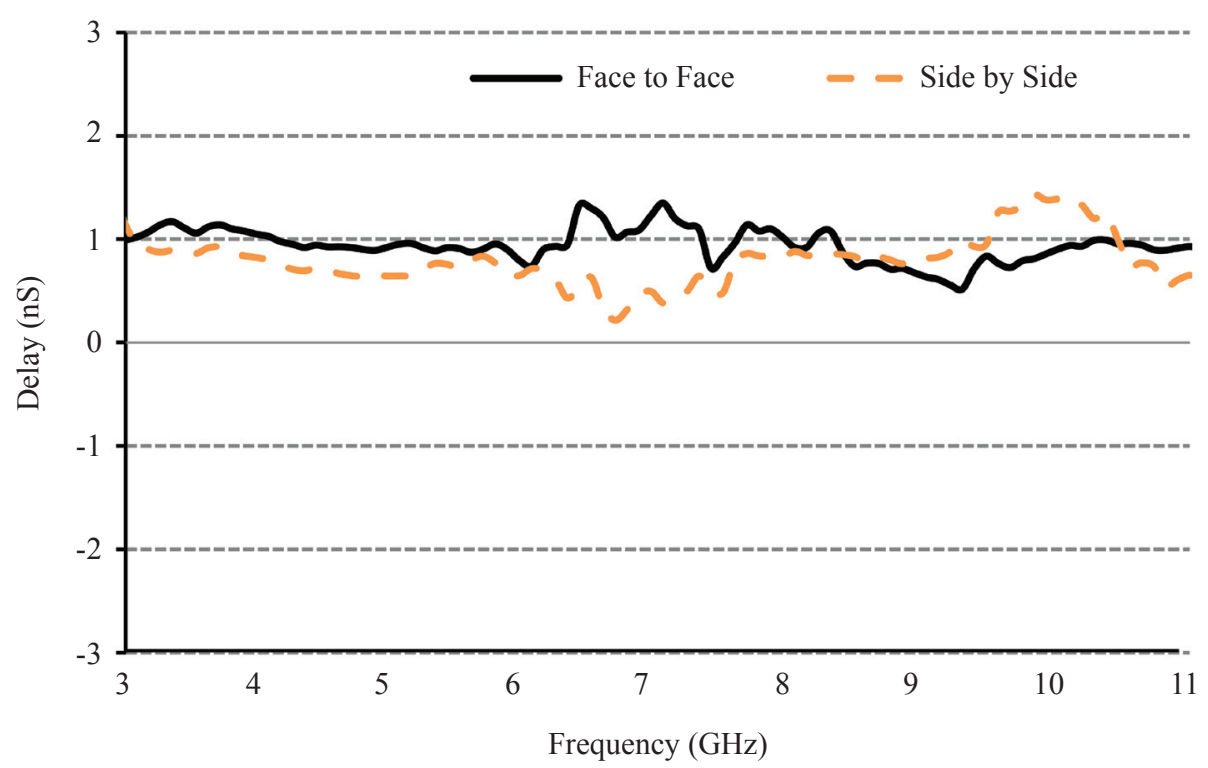

FIGURE 10. Measured group delay at UWB operating band

The group delay, that characterizes the non-linearly of the signal, of the prototype is measured by placing two fabricated antennas at a distance of $0.5 \mathrm{~m}$ apart. For distortion-free transmission of a signal in UWB frequency band, the group delay should be linear and small. From the measured group delay presented in Figure 10, it can be commented that the delay is nearly flat and the average group delay in face-to-face and side-by-side orientation are respectively about $0.95 \mathrm{nS}$ and $0.8 \mathrm{nS}$. These very small variations of group delay ensure that the presented antenna possesses better phase linearity in the whole UWB frequency band.

A comparison of the performance of the proposed antenna with the recently reported antennas is presented in Table 1. It can be observed from the table that the proposed antenna exhibits better performance in terms of fractional bandwidth and gain with smaller size and planar profile.

\section{CONCLUSION}

The design and prototyping of a planar microstrip patch antenna with a dimension of $29 \times 20.5 \mathrm{~mm}^{2}$ is presented in this paper. The defection of ground plane by making it partial one and addition of slots has been employed to enhance the operating bandwidth. Experimental verification shows that the designed antenna is able to achieve a super-wide impedance bandwidth of $15.37 \mathrm{GHz}$ with a fractional bandwidth of $149 \%$, good gain, exhibits stable omnidirectional radiation patterns, and thus suitable to be used in WiMAX, WiFi, S-band, WLAN, UWB, C-band, X-band, Ku-band wireless communication applications. The layout of the designed antenna is very simple and easy to be incorporated in RF circuitry with low manufacturing cost. 
TABLE 1. Comparison of the proposed antenna with recently reported wideband antennas

\begin{tabular}{lccccc}
\hline \multicolumn{1}{c}{ Antennas } & Dimension $(\mathrm{mm})$ & Operating Band & Bandwidth (GHz) & Fractional Bandwidth (\%) & Gain $(\mathrm{dBi})$ \\
\hline (Ghosh et al. 2016) & $40 \times 45$ & $2.9-6.0 \mathrm{GHz}$ & 3.10 & 70 & - \\
(Alsath \& Kanagasabai 2015) & $24 \times 16$ & $3.1-10.9 \mathrm{GHz}$ & 7.90 & 112 & 3.5 \\
(Jiang et al. 2009) & $35 \times 35$ & $2.8-10.7 \mathrm{GHz}$ & 7.90 & 117 & - \\
(Ali et al. 2016) & $41 \times 41$ & $3.6-15 \mathrm{GHz}$ & 11.4 & 123 & 3.11 \\
(Azim et al. 2013b) & $39 \times 40$ & $2.6-12.3 \mathrm{GHz}$ & 9.70 & 130 & 3.67 \\
(Azim et al. 2011c) & $30 \times 22$ & $3.08-15.9 \mathrm{GHz}$ & 12.82 & 135 & 4.04 \\
(Adam et al. 2013) & $30 \times 35$ & $2.1-12 \mathrm{GHz}$ & 9.90 & 140 & $2-6$ \\
Azim et al. 2013a) & $27 \times 23.5$ & $3.3-20 \mathrm{GHz}$ & 16.7 & 143 & 5.11 \\
(This work) & $29 \times 20.5$ & $2.63-18 \mathrm{GHz}$ & 15.37 & 149 & 5.85 \\
\hline
\end{tabular}

ACKNOWLEDGEMENTS

The authors would like to thank the University of Chittagong to sponsor this research work under research grant no.: $88 /$ P\&D/7-35(3)/2017. The authors also thank the Department Electrical, Electronic \& Systems Engineering of Universiti Kebangsaan Malaysia for helping them to use their laboratory facility in measuring the antenna performances.

\section{REFERENCES}

Adam, A. A., Rahim, S. K. A., Tan, K. G. \& Reza, A. W. 2013 Design of 3.1-12 GHz printed elliptical disc monopole antenna with half circular modified ground plane for UWB application. Wireless Personal Communications 69(2): 535-549.

Ali, W. A., Mansour, A. M. \& Mohamed, D. A. 2016. Compact UWB wearable planar antenna mounted on different phantoms and human body. Microwave and Optical Technology Letters 58(10): 2531-2526.

Alsath, M. G. N. \& Kanagasabai, M. 2015. Compact UWB monopole antenna for automotive communications. IEEE Transactions on Antennas and Propagation 63(9): 4204-4208.

Ansari, J. A., Yadav, N. P., Mishra, A., Singh, P. \& Vishvakarma, B. R. 2012. Analysis of multilayer rectangular patch antenna for broadband. Wireless Personal Communications 62(2): 315-327.

Azim, R., Islam, M. T., Misran, N. \& Mobashsher, A. T. 2011b. Compact UWB planar antenna for broadband applications. Informacije MIDEM 41(1): 37-40.

Azim, R., Islam, M. T. \& Misran, N. 2011a. Design of a planar UWB antenna with new band enhancement technique. Applied Computational Electromagnetic Society Journal 26(10): 856-862.

Azim, R., Islam, M. T. \& Misran, N. 2011c. Ground modified double-sided printed compact UWB antenna. Electronics Letters 47(1): 9-11.

Azim, R., Islam, M. T. \& Misran, N. 2013a. Printed circular disc compact planar antenna for UWB applications. Telecommunication Systems 52(2): 1171-1177.
Azim, R., Islam, M. T. \& Misran, N. 2013b. Microstrip line-fed printed planar monopole antenna for UWB applications. Arabian Journal for Science and Engineering 38(9): 2415-2422.

Ghosh, C. K., Mondal, S. \& Parui, S. K. 2016. A compact multiband microstrip antenna using complementary slots on the ground plane. Microwave and Optical Technology Letters 58(1): 47-51.

Hussine, U. U., Islam, M. T. \& Misran, N. 2012. Analysis of Microstrip Patch Antenna for L1 and L2 for Global Positioning System Applications. Jurnal Kejuruteraan (Jounal of Engineering) 24: 29-33.

Jiang, J.-B., Song, Y., Yan, Z.-H., Zhang, X. \& Wu, W. 2009. Band-notched UWB printed antenna with an invertedL-slotted ground. Microwave and Optical Technology Letters 51(1): 260-263.

Li, D., Guo, P., Dai, Q. \& Fu, Y. 2012. Broadband capacitively coupled stacked patch antenna for GNSS applications. IEEE Antennas and Propagation Letters 11: 701-704.

Li, M. \& Behdad, N. 2017. A compact, capacitively fed UWB antenna with monopole-like radiation characteristics. IEEE Transactions on Antennas and Propagation 65(3): 1026-1037.

Lu, Y., Huang, Y., Chattha, H. T. \& Cao, P. 2011. Reducing ground-plane effects on UWB monopole antennas. IEEE Antennas and Propagation Letters 10: 147-150.

Nazli, H., Bicak, E., Turetken, B. \& Sezgin, M. 2010. An improved design of planar elliptical dipole antenna for UWB applications. IEEE Antennas and Propagation Letters 9: 264-267.

Rahman, M. N., Islam, M. T., Mahmud, M.Z. \& Samsuzzaman, M. 2017. Compact microstrip patch antenna proclaiming super wideband characteristics. Microwave and Optical Technology Letters 59(10): 2563-2570.

Reddy, G. S., Mishra, S. K., Kharche, S. U. \& Mukherjee, J. 2012. High gain and low cross-polar compact printed elliptical monopole UWB antenna loaded with partial ground and parasitic patches. Progress in Electromagnetics Research B 43: 151-167.

Saad, R. E., Khaled, E. M. \& Salem, D. A. 2011. Wideband slotted planar antenna with defective ground structure. PIERS 2016, Proceedings 1092-1097. 
Tran, H. H. \& Park, I. 2016. Compact wideband circularly polarised resonant cavity antenna using a single dielectric superstrate. IET Microwaves, Antennas \& Propagation 10(7): 729-736.

*Rezaul Azim

A. K. M. Ariful Haque Siddique

Department of Physics, University of Chittagong

Chittagong 4331, Bangladesh.

*Corresponding author; email: rezaulazim@cu.ac.bd

Received date: $20^{\text {th }}$ January 2018

Accepted date: $9^{\text {th }}$ March 2018

Online First date: $1^{\text {st }}$ September 2018

Published date: $31^{\text {st }}$ October 2018 